\section{ORIGINAL RESEARCH}

\author{
D.A. Lewis \\ Y.H. Ding \\ D. Dai \\ R. Kadirvel \\ M.A. Danielson \\ H.J. Cloft \\ D.F. Kallmes
}

\title{
Morbidity and Mortality Associated with Creation of Elastase-Induced Saccular Aneurysms in a Rabbit Model
}

\begin{abstract}
BACKGROUND AND PURPOSE: Elastase-induced aneurysms in rabbits have been proposed as a useful preclinical tool for device development. The object of this study was to report rates of morbidity and mortality associated with the creation and embolization of elastase-induced rabbit aneurysms and to assess the impact of operator experience on these rates.
\end{abstract}

MATERIALS AND METHODS: Elastase-induced model aneurysms were created in New Zealand white rabbits $(n=700)$. One neuroradiologist/investigator, naive to the aneurysm-creation procedure at the outset of the experiments, performed all surgeries. All morbidity and deaths related to aneurysm creation $(n=700)$ and embolization procedures $(n=529)$ were categorized into acute and chronic deaths. Data were analyzed with single-regression analysis and analysis of variance. To assess the impact of increasing operator experience, we broke the number of animals into 50-animal increments.

RESULTS: There were 121 (17\%) deaths among 700 subjects. Among 700 aneurysm-creation procedures, 59 deaths $(8.4 \%)$ were noted. Among 529 aneurysm-embolization procedures, 43 deaths (8.1\%) were noted. Nineteen additional deaths ( $2.7 \%$ of 700 subjects) were unrelated to the procedures. Simple regression-indicated mortality associated with procedures diminished with increasing operator experience $\left(R^{2}=0.38, P=.0180\right)$, and that for each 50-rabbit increment mortality was reduced, on average, by $0.6 \%$.

CONCLUSIONS: Mortality rates of approximately $8 \%$ are associated with both experimental aneurysm creation and with embolization in the rabbit elastase-induced aneurysm model. Increasing operator experience is inversely correlated with mortality, and the age of the rabbit is positively associated with morbidity.

$\mathbf{T}$ he development of endovascular occlusion devices has been facilitated through the use of various animal models of saccular aneurysms. Detachable platinum coils have been tested by using surgically created aneurysms in swine, canines, rabbits, and primates. ${ }^{1-7}$ More recently, elastase-induced aneurysms in rabbits have been proposed as a useful preclinical tool for device development. ${ }^{8-14}$

Previous studies ${ }^{9,10,15}$ have shown that elastase-induced aneurysms in rabbits are similar to human aneurysms in size and shape. A recent study of the histopathology of the rabbit model ${ }^{16}$ has shown that it mimics well the healing seen in human aneurysms, especially when compared with the swine model. Furthermore, because these experimental aneurysms are in close proximity to the aorta and are situated on a curved vessel, they also are exposed to substantial hemodynamic stress.

We report rates of morbidity and mortality associated with model aneurysm creation and correlate these rates with operator experience and type of procedure.

Received May 14, 2008; accepted after revision July 2.

From the Neuroradiology Research Laboratory, Department of Radiology, Mayo Clinic, Rochester, Minn

This work was funded in part by National Institutes of Health Grants NS42646 and HL72247.

Please address correspondence to Debra A. Lewis, PhD, Mayo Clinic, 200 First St SW, Rochester, MN 55905; e-mail: lewis.debra@mayo.edu

Indicates open access to non-subscribers at www.ajnr.org

DOI 10.3174/ajnr.A1369

\section{Materials and Methods}

All animals used in this study underwent surgeries that were approved by the Animal Care and Use Committee of our institution. Seven hundred female white New Zealand rabbits were used in this retrospective study. The single investigator who performed all the surgeries was a trained neuroradiologist who was naive to the aneurysm-creation procedure at the outset of the experiments. All the rabbits used in this study were originally used as part of another investigation. The original investigation was entirely unrelated to this project.

\section{Aneurysm Creation}

The surgical procedures used to create an aneurysm have been described elsewhere. ${ }^{9,17}$ Briefly, female New Zealand white rabbits (2-4 kg) were anesthetized with ketamine $(74 \mathrm{mg} / \mathrm{kg})$, xylazine $(5 \mathrm{mg} / \mathrm{kg})$, and acepromazine $(1 \mathrm{mg} / \mathrm{kg})$. Using a sterile technique through a vertical midline incision in the neck, the trachea and the right carotid artery were identified. The vagus nerve and jugular vein were gently dissected free from the right common carotid artery (CCA). A 5F sheath (Cordis Endovascular, Miami Lakes, Fla) was advanced retrograde in the CCA to a point approximately $3 \mathrm{~cm}$ cephalad to the CCA origin. Through this indwelling sheath, a 3F Fogarty balloon (Baxter Healthcare, Irvine, Calif) was advanced to the origin of the right CCA at its junction with the subclavian artery. The balloon was inflated with iodinated contrast with just enough pressure to achieve flow arrest in the CCA. Porcine elastase (5.23 U/mgP, $40.1 \mathrm{mgP} / \mathrm{mL}$, approximately $200 \mathrm{U} / \mathrm{mL}$; Worthington Biochemical, Lakewood, NJ) mixed with saline and iodinated contrast was incubated in the dead space of the CCA above the inflated balloon for approximately 20 minutes. Following incubation of the elastase solution, the balloon and sheath were removed, the CCA was ligated below the sheath entry site, and the incision was closed in 2 layers. The fascial layers 
were closed with interrupted 4-0 Vicryl (Ethicon, Somerville, NJ) suture. Interrupted subcuticular sutures as well as running skin sutures, both Vicryl, were used to close the wound.

\section{Embolization Procedure}

There was a minimum of 21 days between the aneurysm creation ${ }^{10}$ and the embolization. The animal was prepared for the embolization procedure by administration of the same dose of cocktail as in aneurysm creation, with an intramuscular injection of ketamine/xylazine/ acepromazine followed by maintenance anesthesia with ketamine and xylazine. With sterile technique, surgical exposure of the right common femoral artery was performed. The artery was ligated disvanced retrograde into the artery. A 0.018-inch guidewire was passed through the angiocatheter, followed by placement of a $5 \mathrm{~F}$ vascular sheath. Heparin $(100 \mathrm{U} / \mathrm{kg})$ was administered intravenously. As described previously, ${ }^{8}$ a $5 \mathrm{~F}$ catheter (Envoy; Cordis Neurovascular Systems, Miami Lakes, Fla) was advanced into the brachiocephalic artery. With a coaxial technique, with continuous heparinized saline flush, a 2-marker microcatheter was advanced into the aneurysm cavity. The size of the aneurysm cavity was assessed by using direct comparison with radiopaque sizing devices during digital subtraction angiography (DSA). Where possible, maximal packing of devices at the parent artery/neck interface was achieved. Following the embolization, a final control DSA was performed. The catheters and sheath were removed, the femoral artery was ligated, and the incision was closed, as in the aneurysm creation.

\section{Dataset}

The 700 animals with surgeries, aneurysm creations, and embolizations, spanning 4 years, were subdivided into groups of 50 . Within each group of 50, 2 categories were created, including those who survived until their scheduled sacrifice and those who did not. Those who did not survive were further subdivided into categories describing the cause of death.

\section{Statistics}

Analysis of variance (JMP; SAS, Cary, NC) coupled with Student $t$ post hoc tests, when necessary, was used to determine if there were any differences between any of the categories/causes of death. Correlations (JMP, SAS) were run on the number of incidents in the category in question by the number of creations in 50-animal increments. Finally, multiple regression analyses (JMP, SAS) were run on all the independent variables within each group (acute and chronic aneurysm creation and acute and chronic embolization) to determine which variable was the largest contributor to the $R^{2}$ value and if there were additive effects with more than 1 independent variable.

\section{Results}

There were 121 (17\%) deaths among 700 subjects, which reflected a total of 1259 procedures (700 aneurysm-creation procedures and 529 embolizations). Causes of death included (Table) anesthesia-related deaths; device failure; failure to thrive (FTT), which included animals that would not eat after surgery and became lethargic; self-mutilation, which included animals that chewed their incisions or, owing to an injection too close to the sciatic nerve, chewed their foot; paralysis; unknown causes of death; and other, which included those that we believed were strokes, which we were unable to document fully. Unrelated to the procedures, we sacrificed 12 subjects tally by using $4-0$ silk suture, and a 22-gauge angiocatheter was ad-

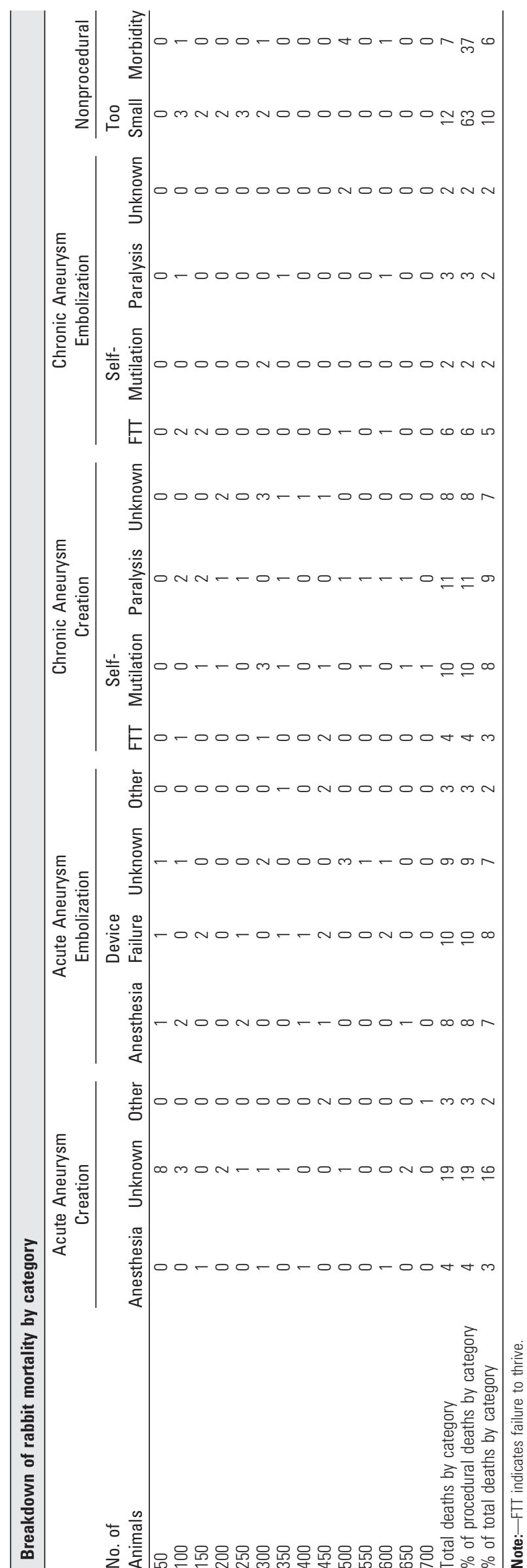




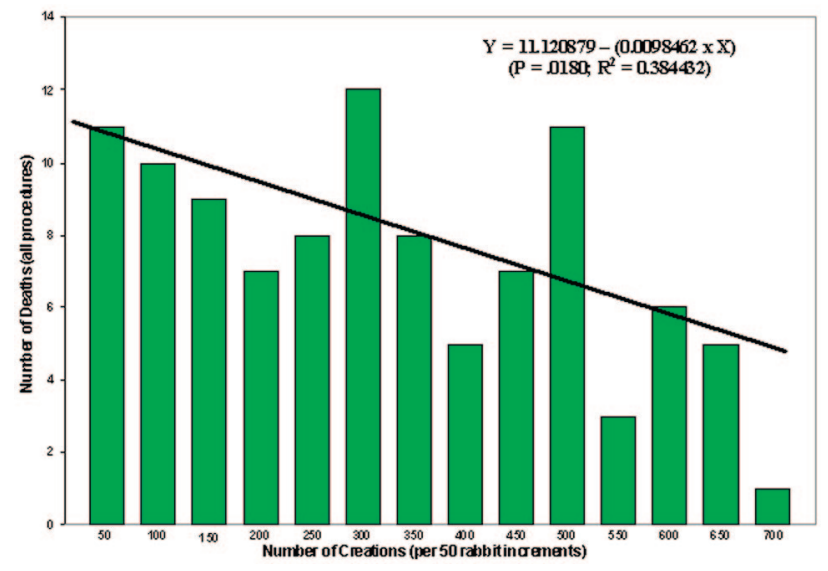

Fig 1. Bar chart shows the number of deaths due to all procedures (aneurysm creation and embolization) over 50-rabbit increments. The line indicates the linear regression for these data. In addition, the linear regression equation $[Y=11.120879-(0.0098462 \times X)]$ is noted along with the $P$ value $(P=.0180)$ and $R^{2}\left(R^{2}=0.384432\right)$ value for the equation.

because of unfavorable aneurysm morphology, such as being too small to embolize, and 7 animals because of morbidities such as hairballs, a lacerated liver, a corneal ulcer, and a gastric ulcer.

The 2 categories related to procedural deaths, aneurysm creation, and embolization were divided into acute, at the time of surgery, and chronic postsurgical complications. Of the 121 deaths, 102 were procedure-related and 19 were not related to the procedure. Fifty-nine (58\% of 102 procedure-related deaths) of the procedure-related deaths were associated with both acute and chronic aneurysm creation, yielding a mortality rate of $8.4 \%$ for aneurysm-creation procedures. Fortythree ( $42 \%$ of 102 procedure related deaths) were related to both acute and chronic aneurysm embolization, yielding a mortality rate of $8.1 \%$ for aneurysm embolization procedures.

A breakdown of all deaths by category (creation, embolization, and nonprocedural deaths) showed that the single greatest cause of death was aneurysm creation. If deaths due to aneurysm creation are plotted for 59 rabbit increments, the data show that the number of deaths due to aneurysm creation are diminished by more than half after 100 creations and remain fairly constant thereafter, regardless of the time between surgeries.

A Pearson product moment correlation (JMP, SAS) showed a significant $\left(P=.0246, R^{2}=0.354738\right)$ negative relationship between the number of aneurysm-creation deaths and the number of creations in 50-rabbit increments. If all procedural deaths (aneurysm creations and embolizations) are correlated with the number of creations, the significant negative relationship remains $\left(P=.0180, R^{2}=0.384432\right.$; Fig $1)$. When the number of days between the start of each 50 surgery increment and the finish of that increment was examined in relation to the same factors, there were no significant correlations. Finally, the factor "unknown cause of death" was the single largest contributor to the multiple regression equations for both the acute $\left(P=.301, R^{2}=0.3352\right)$ and chronic $\left(P=.0279, R^{2}=0.3424\right)$ aneurysm-creation equations as well as for the acute $\left(P=.0471, R^{2}=0.2896\right)$ embolization equation. The single greatest contributor to the chronic embolization equation was the FTT variable $\left(P=.0175, R^{2}=0.3873\right)$.

The FTT group, as defined previously, was the group that was unable to return to normal eating habits, etc after embolization. When the average age of these animals was compared with the average age of the other animals that had embolization procedures, it was discovered that the FTT animals were significantly older $(591 \pm 362$ days $)$ than the other animals (159 \pm 298 days, $P=.0004)$.

\section{Discussion}

The rabbit model of elastase-induced aneurysm creation is being used by multiple research laboratories. ${ }^{18-22}$ With more laboratories trying to use the model come anecdotal reports of high mortality/morbidity rates and 1 published report ${ }^{23}$ of a $54 \%$ death rate. This current retrospective study shows an approximately $8 \%$ death rate for both aneurysm-creation procedures and embolization procedures. Furthermore, we noted that as might be expected, mortality/morbidity rates are inversely related to the number of surgeries performed per operator. These current data suggest that the rabbit model can be applied in experienced hands with low mortality but that a learning curve is present. These data are important in guiding researchers new to the technique, regarding expected numbers of total subjects that will be needed to complete a desired sample size. The data may also inform researchers that their own mortality rates may be excessive, as noted previously, and that low rates are indeed attainable.

Multivariate analysis of variance showed that the only factor that correlated with both acute and chronic aneurysm-creation deaths was what was termed "unknown causes of death" (19 of 36 acute creation deaths and 8 of 33 chronic creation deaths). We suspect that most of these deaths were due to stroke. However, conclusive evidence for this diagnosis was not available, thus, the unknown category was created. The reason why the aneurysm-creation surgery may be more conducive to stroke can only be based on conjecture. The surgery requires a complete block of flow to the brain in the right carotid artery. Although the rabbit does have a circle of Willis, it may be that there is some biologic variability in the adequacy of the rerouted flow in some of the animals.

Acute and chronic embolization deaths accounted for $42 \%$ of the 121 total deaths. Multivariate analysis showed that the factors making an impact on acute embolization deaths were unknown causes of death ( 9 of 30) and also device failure (10 of 30). Factors making a significant impact on chronic embolization deaths were failure to thrive (6 of 13) and paralysis ( 3 of 13). Most interesting, the average time from embolism creation until sacrifice in the FTT group was significantly older $(P=.0004)$ than the other animals that had embolization procedures. This indicates that the younger the animals are, the better chance they have to survive chronic embolization.

One study (Thiex et $\mathrm{al}^{23}$ ) cites hemorrhagic tracheal necrosis as a leading cause of death ( 9 of 24 rabbits). In the Thiex study, the authors found an arterial branch coming off the CCA and providing blood to the trachea. When this branch was accommodated, the authors had no further complications due to tracheal necrosis. Although this issue was not a problem in our study, it does point out the need to be mindful of anatomic variations, not only in the creation phase but throughout any treatment phase as well.

The fact that the exact cause of death was not known in 
every case was 1 limitation in this study. The other limitation was the fact that though 1 operator enabled us to provide a unique examination of changes in death rates with time, it also means that we cannot say that every operator will have diminishing mortality rates during the same time period.

\section{Conclusions}

Mortality rates of approximately $8 \%$ are associated with both experimental aneurysm creation and with embolization in the rabbit elastase-induced aneurysm model. Increasing operator experience is inversely correlated with mortality, and the age of the rabbit is positively associated with morbidity.

\section{References}

1. Reul J, Weis J, Spetzger U, et al. Long-term angiographic and histopathologic findings in experimental aneurysms of the carotid bifurcation embolized with platinum and tungsten coils. AJNR Am J Neuroradiol 1997;18:35-42

2. Bocher-Schwarz $\mathrm{H}$, Ringel $\mathrm{K}$, Bohl J, et al. Histological findings in coil-packed experimental aneurysms 3 months after embolization. Neurosurgery 2002;50: 379-84, discussion 384-85

3. Guglielmi G, Ji C, Massoud T, et al. Experimental saccular aneurysms. II. A new model in swine. Neuroradiology 1994;36:547-50

4. Graves V, Partington C, Rufenacht D, et al. Treatment of carotid artery aneurysms with platinum coils: an experimental study in dogs. AJNR Am J Neuroradiol 1990;11:249-52

5. Graves V, Strother C, Rappe A. Treatment of experimental canine carotid aneurysms with platinum coils. AJNR Am J Neuroradiol 1993;14:787-93

6. Mawad M, Mawad J, Cartwright JJ, et al. Long-term histopathologic changes in canine aneurysms embolized with Guglielmi detachable coils. AJNR Am J Neuroradiol 1995;16:7-13

7. Tenjin H, Fushiki S, Nakahara Y, et al. Effect of Guglielmi detachable coils on experimental carotid artery aneurysms in primates. Stroke 1995;26:2075-80

8. Kallmes D, Helm G, Hudson S, et al. Histologic evaluation of platinum coil embolization in an aneurysm model in rabbits. Radiology 1999;213:217-22

9. Altes TA, Cloft HJ, Short JG, et al. 1999 ARRS Executive Council Award: creation of saccular aneurysms in the rabbit-a model suitable for testing endovascular devices. American Roentgen Ray Society AJR Am J Roentgenol 2000;174:349-54
10. Fujiwara N, Cloft H, Marx W, et al. Serial angiography in an elastase-induced aneurysm model in rabbits: evidence for progressive aneurysm enlargement after creation. AJNR Am J Neuroradiol 2001;22:698-703

11. de Gast A, Altes T, Marx W, et al. Transforming growth factor beta-coated platinum coils for endovascular treatment of aneurysms: an animal study. Neurosurgery. 2001;49:690-94

12. Marx W, Cloft H, Helm G, et al. Endovascular treatment of experimental aneurysms by use of biologically modified embolic devices: coil-mediated intraaneurysmal delivery of fibroblast tissue allografts. AJNR Am J Neuroradiol 2001;22:323-33

13. Kallmes D, Fujiwara N. New expandable hydrogel-platinum coil hybrid device for aneurysm embolization. AJNR Am J Neuroradiol 2002;23:1580-88

14. Kallmes D, Fujiwara N, Yuen D, et al. A collagen-based coil for embolization of saccular aneurysms in a New Zealand white rabbit model. AJNR Am J Neuroradiol 2003;24:591-96

15. Short J, Fujiwara N, Marx W, et al. Elastase-induced saccular aneurysms in rabbits: comparison of geometric features with those of human aneurysms. AJNR Am J Neuroradiol 2001;22:1833-37

16. Dai D, Ding Y, Danielson $M$, et al. Histopathologic and immunohistochemical comparison in human, rabbit, and swine aneurysms embolized with platinum coils. AJNR Am J Neuroradiol 2005;26:2560-68

17. Ding Y, Danielson M, Kadirvel R, et al. Modified technique to create morphologically reproducible elastase-induced aneurysms in rabbits. Neuroradiology 2006;48:528-32

18. Onizuka M, Miskolczi L, Gounis $M$, et al. Elastase-induced aneurysms in rabbits: effect of postconstruction geometry on final size. AJNR Am J Neuroradiol 2006;25:1129-31

19. Krings T, Hans F, Moller-Hartmann W, et al. Treatment of experimentally induced aneurysms with stents. Neurosurgery 2005;56:1347-59

20. Yasuda H, Kuroda S, Nanba R, et al. A novel coating biomaterial for intracranial aneurysms: effects and safety in extra- and intracranial carotid artery. Neuropathology 2005;25:66-76

21. Hans F, Moller-Hartmann W, Brunn A, et al. Treatment of wide-necked aneurysms with balloon-expandable polyurethane-covered stentgrafts: experience in an animal model. Acta Neurochir (Wein) 2005;147:871-76. Epub 2005 Mar 17

22. Doerfler A, Becker W, Wanke I, et al. Multimodal imaging in the elastaseinduced aneurysm model in rabbits: a comparative study using serial DSA, MRA and CTA. Rofo 2004;176:590-96

23. Thiex R, Hans F, Krings T, et al. Haemorrhagic tracheal necrosis as a letha complication of an aneurysm model in rabbits via endoluminal incubation with elastase. Acta Neurochir (Wien) 2004;146:285-89, discussion 289. Epub 2004 Jan 22 